\title{
[gw22-e0346] STUDY OF ASSESSMENT OF 18F-FDG IN APOE-/- MICE ATHEROSCLEROTIC PLAQUES BY AUTORADIOGRAPHY
}

Wang Xiaona, Liu Hongbin, Zhang Jinming, Li Jian, Wang GuoweiChinese Pla General Hospital, Beijing, China

10.1136/heartjnl-2011-300867.177

Objective To investigate the possibility of application of nuclide on the $\mathrm{ApoE}^{-/-}$mice plaque, we used ${ }^{18} \mathrm{~F}-\mathrm{FDG}$ to image the plaque and elucidate the correlation between the nuclear imaging and histological imaging, estimate the feasibility of ${ }^{18} \mathrm{~F}-\mathrm{FDG}$ on detecting vulnerable plaque.

Methods Eight-week-old male ApoE ${ }^{-/-}$mice were fed with western diet (was provided by academy of military medical sciences) till 44 weeks as experimental group. After fasting for (10 to 12$) \mathrm{h}, 0.56$ to $0.71 \mathrm{mCi}{ }^{18} \mathrm{~F}-\mathrm{FDG}$ was injected into the mice tail vein. At $30 \mathrm{~min}$ after injection of the radiotracer, we sacrificed the mice and removed the aortas, incised longitudinally. Aortas were weighted, and radioactivity was measured with a well-type $\gamma$-counter, the results were expressed as the SUV. Then macroautoradiographies were acquired, aorta plaque and macrophage were investigated by examination of stained sections by Red-O staining and CD68 staining. Harvestings of aortas for en face analysis were performed with oil Red-O to compare with atuoradiography, and CD68 staining to compare with SUV. The control group C57BL/6N mice were fed with full diet, and the rest work were alike.

Results There was a significant difference $(0.243 \pm 0.054$ vs $0.112 \pm 0.004, t=4.108, p=0.002)$ in the uptake of ${ }^{18} \mathrm{~F}-\mathrm{FDG}$ between experimental group and control group. Control group were negative result. Macrophage (Mf) number between experimental group and control group was significant different $(4.99 \pm 0.51$ vs $9.87 \pm 0.31, t=2.263, p=0.037)$, and the ${ }^{18} \mathrm{~F}$ FDG uptake and macrophage (Mf) number in thoracic aortic segments had a strong correlation ( $r=0.835, p=0.0002)$, but the en face measurements of aortas isolated 30 min after ${ }^{18} \mathrm{~F}$ FDG injections (5.848 $\pm 2.416: 1)$ demonstrated a weaker correlation between fat stainings and autoradiographies $(r=0.4697$, $\mathrm{p}=0.001$ ). Macrophage (Mf) number in visualisation plaque was significantly different from the unvisualisation plaque (4.29 \pm 0.42 vs $10.85 \pm 1.47, t=10.55, p=0.015)$.

Conclusions The uptake of ${ }^{18}$ F-FDG in aorta plaque was enhanced and had higher specificity than non-target tissue. The uptake of ${ }^{18}$ F-FDG had a strong correlation with Mf number, but the correlation between SUV and the en face of plaque is weak. 